\title{
Insoluble poly(anthranilic acid) confined in Nafion membrane by chemical and electrochemical polymerization of anthranilic acid
}

\author{
S. Patra, N. Munichandraiah* \\ Department of Inorganic and Physical Chemistry, Indian Institute of Science, Bangalore 560012, India
}

\begin{abstract}
Self-doped polyaniline (PANI) possesses superior electrochemical properties and processability in relation to the PANI due to an acid group substituted on the polymer backbone. However, the polar acid group causes the self-doped PANI to undergo dissolution in aqueous, in particular, acidic solutions. To prevent the solubility, poly(anthranilic acid), PANA, is confined in the cavities of the Nafion membrane by a novel electrochemical as well as a conventional chemical polymerization of anthranilic acid. The PANA is characterized by electrochemical, optical, spectroscopic and scanning electron microscopic studies.
\end{abstract}

Keywords: Anthranilic acid; Polymerization; Poly(anthranilic acid); Cyclic voltammetry; UV-visible spectroscopy

\section{Introduction}

Polyaniline (PANI) has been extensively studied for a variety of its chemical, optical, electrical, electrochemical and electrochromic properties owing to its ease of synthesis and a wide range of applications [1]. PANI can be synthesized conveniently either as a powder or as a film by chemical and electrochemical routes, and it is stable in air, humid conditions and also in dilute acidic solutions. However, PANI is insoluble in most of the aqueous and non-aqueous solvents, thus restricting its use only as the solid. For the purpose of polymer processing, PANI solutions are essential and one of the approaches reported in the literature to dissolve is introducing an acid group in its structure [2]. For instance, sulfonic acid group has been substituted to hydrogen on the benzene ring of PANI, resulting in a self-doped PANI [3]. A post-sulfonation of PANI makes the resulting polymer soluble. Also, polymerization of 2-amino benzene sulfonic acid (metanilic acid) in acidic aqueous solutions produces soluble poly(metanilic acid) [4]. Thus, preparation of a solid polymer of this type is not possible in aqueous acidic solutions, but

\footnotetext{
* Corresponding author. Tel.: +91 802293 3183; fax: +91 8023600683

E-mail address: muni@ipc.iisc.ernet.in (N. Munichandraiah).
}

it may be possible in a neutral solution of aqueous-organic mixed medium [5]. However, in order to exhibit the electrical and electrochemical properties, protonation of the imine nitrogen of PANI backbone in poly(metanilic acid) is essential, which requires an acidic solution [6]. Thus, the insolubility of PANI on one hand and the solubility of acid group substituted PANI on the other make the polymer system complex. An acid group substituted, self-doped PANI is expected to possess better electrical and electrochemical properties over wider $\mathrm{pH}$ range if it is available in solid form than the unsubstituted PANI. Thus, the synthesis of an acid group substituted solid PANI from the corresponding monomer in acidic solutions is a challenging problem.

Anthranilic acid (2-amino benzoic acid) is an important monomer for the synthesis of carboxylic acid group substituted PANI. Similar to the poly(metanillic acid) [7], it is expected that poly(anthranilic acid), PANA, possesses electrochemical activity over a wide $\mathrm{pH}$ range in aqueous solutions owing to the substitution of carboxylic acid group. Studies on the synthesis of PANA from acidic aqueous solutions are scarcely reported in the literature, probably due to the problem of its high solubility. Copolymer of aniline and anthranilic acid has been prepared by chemical polymerization to improve the solubility of PANI, study 
the self-doping mechanism and evaluate thermal properties [8]. Also, this study involves chemical polymerization of anthranilic acid in an alkaline solution [8]. However, the soluble PANA homopolymers (with or without external dopant $\mathrm{HCl}$ ) exhibit very low conductivity compared to PANI. For the copolymers, the solubility increases while the conductivity decreases with the amount of anthranilic acid in the monomers. Also, chemical polymerization of anthranilic acid in diluted $\mathrm{HCl}$ results in the formation of PANA with very low yield due to its high solubility [8]. In another study [9], films of copolymers of aniline and anthranilic acid have been grown on gold electrodes by applying cyclic potential sweeps. A combined diffused reflectance spectroelectrochemistry with quartz crystal microbalance has been shown to be a useful technique to acquire multidimensional information during the growth of the copolymer films. These studies clearly suggest that preparation of PANA homopolymer in solid state is difficult because of its solubility.

In the present study, solid PANA is synthesized in an acidic medium from a chemical route and also a novel electrochemical route. The solid polymer thus formed is confined in the porous matrix of the Nafion membrane and it does not dissolve in acidic solutions. The PANI backbone of the PANA exhibits enhanced electrochemical activity due to self-doping of carboxylic acid group in electrolytes of a wide $\mathrm{pH}$ range, in relation to PANI.

\section{Experimental}

Analytical grade chemicals-anthranilic acid, $\mathrm{H}_{2} \mathrm{SO}_{4}$, $\mathrm{Na}_{2} \mathrm{SO}_{4}, \mathrm{NaOH}$ and $\mathrm{NH}_{4} \mathrm{~S}_{2} \mathrm{O}_{8}$ were used as received. Double distilled water was used for the preparation of all solutions. Nafion 112 membrane (thickness $\sim 50 \mu \mathrm{m}$ ) was treated in boiling $\mathrm{H}_{2} \mathrm{O}_{2}, \mathrm{H}_{2} \mathrm{SO}_{4}$ and double distilled water before using for the experiments. A solution of PANA was prepared by dissolving $0.1 \mathrm{M}$ anthranilic acid in $0.5 \mathrm{M} \mathrm{H}_{2} \mathrm{SO}_{4}$ and dropwise addition of $0.1 \mathrm{M} \mathrm{NH}_{4} \mathrm{~S}_{2} \mathrm{O}_{8}$ solution. For the purpose of encapsulation of PANA in the Nafion, a strip ( $35 \mathrm{~mm} \times 5 \mathrm{~mm})$ of the Nafion membrane was soaked in $0.1 \mathrm{M}$ anthranilic acid $+0.5 \mathrm{M} \mathrm{H}_{2} \mathrm{SO}_{4}$ for about $2 \mathrm{~h}$. During this period, anthranilic acid entered the cavities of the Nafion membrane. Then, the film was taken out, rinsed with double distilled water and soaked in $0.1 \mathrm{M} \mathrm{NH}_{4} \mathrm{~S}_{2} \mathrm{O}_{8}+0.5 \mathrm{M} \mathrm{H}_{2} \mathrm{SO}_{4}$ for about $6 \mathrm{~h}$. The anthranilic acid present in the Nafion membrane underwent oxidation producing PANA, which was indicated by the change in colour of the membrane.

For electrochemical polymerization, the anthranilic acid loaded Nafion membrane was rinsed with distilled water, wiped with filter paper to make the surface dry and the electrode assembly was made by sandwiching the Nafion membrane between two Pt foils ( $25 \mathrm{~mm} \times 5 \mathrm{~mm}$ each) as shown in Fig. 1( $a$ and b). A small portion (5-10 mm length marked (i) in Fig. 1(b)) was left un-sandwiched and a major portion of the film (20-25 mm length marked (ii) in Fig. 1(b)) was held in between the Pt foils. The Pt foils were electrically shorted

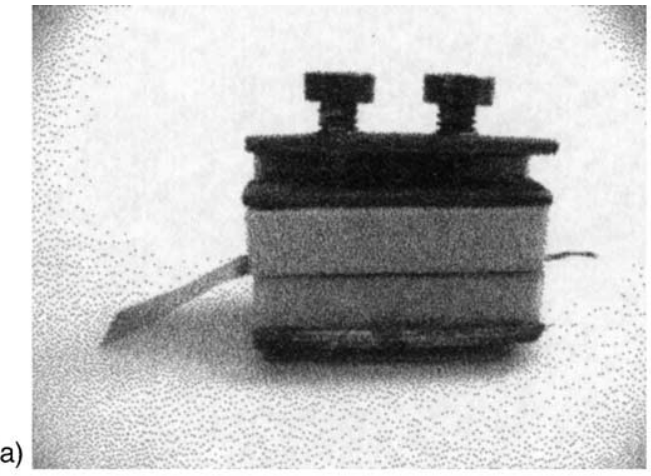

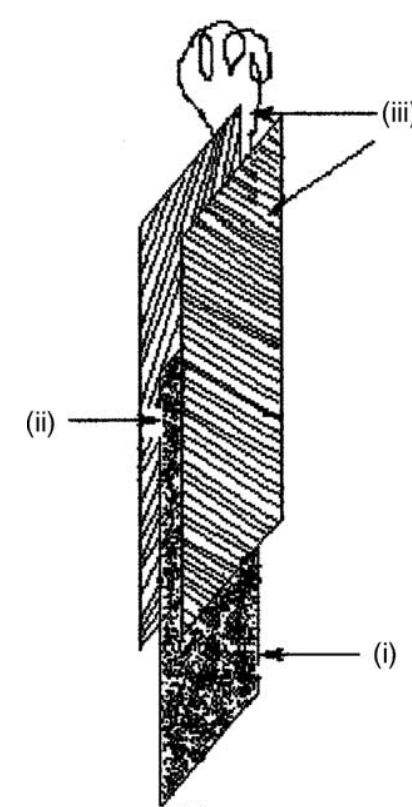

(b)

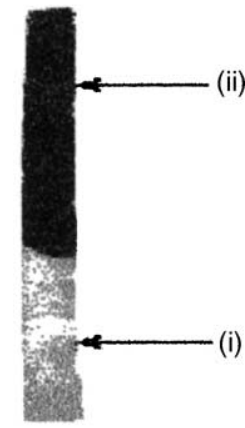

(c)
Fig. 1. (a) A photograph of working electrode assembly of the Nafion membrane sandwiched between two Pt foils and held tightly using Teflon blocks and a clamp; (b) schematic of electrode assembly with Nafion membrane between Pt foils: (i) the portion of Nafion dipped in the electrolyte, (ii) the portion sandwiched between Pt foils and (iii) two Pt foils; (c) a photograph of the Nafion membrane after electrochemical polymerization: (i) the portion dipped in the electrolyte $\left(0.5 \mathrm{M} \mathrm{H}_{2} \mathrm{SO}_{4}\right)$ and (ii) the electrochemically polymerized part.

by means of a copper wire and they were held tightly with the membrane by means of thick insulating supports made of Teflon and a clamp (Fig. 1(a)). This Nafion-Pt working electrode (WE) assembly was introduced into an electrochemical cell in such a way that the un-sandwiched part of the Nafion membrane alone made contact with the liquid electrolyte. Care was taken to avoid the contact of the Pt foils with the solution. The electrochemical cell was made of glass, which had provision to introduce the Nafion-Pt WE assembly, Pt foil counter electrode and a saturated calomel reference electrode (SCE). Potential values are reported against SCE. An electrolyte of $0.5 \mathrm{M} \mathrm{H}_{2} \mathrm{SO}_{4}$ was taken in the cell.

A computer controlled potentiostat/galvanostat EG\&G PARC model Versastat was used for electrochemical experiments. UV-vis and FT-IR spectra were recorded 
by Perkin-Elmer model Lambda 35-UV/VIS spectrometer and Bruker FT-IR model 66V spectrometers, respectively. Scanning electron micrographs (SEM) were recorded by JOEL microscope model JSM-840A.

\section{Results and discussion}

Similar to the oxidation of aniline, the oxidation of anthranilic acid to PANA occurs according to the reaction,

$$
\begin{aligned}
& n \mathrm{C}_{6} \mathrm{H}_{4}(\mathrm{COOH}) \mathrm{NH}_{2} \\
& \quad \rightarrow-\left(-\mathrm{C}_{6} \mathrm{H}_{3}(\mathrm{COOH}) \mathrm{NH}-\right)_{n}-+2 n \mathrm{H}^{+}+2 n \mathrm{e}^{-}
\end{aligned}
$$

The oxidation can occur by a suitable oxidizing agent in a chemical route, and also in electrochemical routes employing galvanostatic, potentiostatic and potentiodynamic methods. It is believed that the potentiodynamic method produces PANI films of better quality than those obtain by galvanostatic and potentiostatic methods [10]. It was attempted to examine the electrochemical preparation of a film of PANA on a Pt electrode in $0.5 \mathrm{M} \mathrm{H}_{2} \mathrm{SO}_{4}$ consisting of $0.1 \mathrm{M}$ anthranilic acid. It was found that there was no solid film produced on the Pt anode even after a continuous electrolysis for extended periods using different electrochemical techniques. During the potentiodynamic cycling of the Pt electrode in the monomer solution, the cyclic voltammograms consisted of current peaks corresponding to the oxidation of anthranilic acid. However, there was no increase in the cyclic voltammetric charge in contrast to an increasing charge during the growth of PANI in aniline solutions [11]. Additionally, the surface of the Pt electrode remained bright suggesting that there was no polymer film formed on it. But the electrolyte slowly changed to brown colour with continuation of electrolysis. It was also attempted to oxidize the monomer in the above electrolyte by adding $\mathrm{NH}_{4} \mathrm{~S}_{2} \mathrm{O}_{8}$ as the oxidizing agent. While there was the formation of a soluble polymer, formed as noted from the change in colour of the solution and also from UV-visible spectroscopy as described below, there was no solid polymer precipitated. It was therefore inferred that the PANA has high solubility in $0.5 \mathrm{M} \mathrm{H}_{2} \mathrm{SO}_{4}$ and it cannot be made in the solid form either as a powder or as a film on the anode. In contrast to these observations, a brownish-black powder has been reportedly obtained in a $\mathrm{HCl}$ solution of $\mathrm{pH}$ $0.2-0.5$ after a prolonged reaction time, apparently with very low yield [8]. This powder was soluble in several organic solvents and aqueous $\mathrm{NaOH}$ to give reddish-brown solutions. It is inferred that a saturated solution of PANA would have exhibited a slow precipitation resulting in the formation of some powder. Nevertheless, it is understood that PANA is highly soluble in aqueous solutions.

There has been interest on Nafion-conducting polymer composites in which the ionomer, besides behaving as a host network for the conducting polymer, provides doping anions and protons, which are essential for electronic conductivity [12]. Several methods have been reported for the preparation of Nafion-conducting polymer composites [13-15]. Electrochemical synthesis has been carried out by electropolymerization of the appropriate monomer on Nafion-modified electrode [13-15]. Alternately, electrolysis of solutions containing both Nafion and the monomer has also been reported [16]. Chemical syntheses of conducting polymers in Nafion are expected to provide membranes with better mechanical properties, since it employs well-defined commercial Nafion membranes [15,17]. The loading of the conducting polymer has been achieved either (i) by soaking the Nafion membrane successively in solutions of the monomer and an oxidant or (ii) by exposing one face of the membrane to the monomer solution and the other to an oxidant solution. While these studies involve loading of insoluble conducting polymer such as PANI, encapsulation of soluble conducting polymer such as PANA in Nafion membranes has not been reported to the knowledge of authors. For loading of solid PANA by chemical polymerization, a piece of Nafion 112 membrane was soaked in $0.5 \mathrm{M} \mathrm{H}_{2} \mathrm{SO}_{4}$ consisting of $0.1 \mathrm{M}$ anthranilic acid for about $2 \mathrm{~h}$ followed by oxidation as detailed in Section 2. During the course of this polymerization process, the Nafion membrane gradually darkened to brown colour. A PANA-Nafion membrane was soaked in $0.5 \mathrm{M} \mathrm{H}_{2} \mathrm{SO}_{4}$ for a few days and it was found that the intensity of brown colour continued to be the same throughout. It was inferred that the PANA was tightly held with in the pores of the Nafion membrane probably by chemical interaction between $-\mathrm{COOH}$ group of PANA and the Nafion structure. This could have prevented dissolution of PANA in $0.5 \mathrm{M} \mathrm{H}_{2} \mathrm{SO}_{4}$ solution.

For electrochemical polymerization, an anthranilic acid loaded Nafion membrane was sandwiched between two Pt foils (Fig. 1(a and b)) as detailed in Section 2. The polymerization was carried out by fixing the WE potential at $1.2 \mathrm{~V}$ or by passing a constant current of $5 \mathrm{~mA} \mathrm{~cm}^{-2}$. Alternatively, the electrode was cycled between -0.2 and $1.2 \mathrm{~V}$ repeatedly at a sweep rate of $20 \mathrm{mV} \mathrm{s}^{-1}$. After several sweeps, the WE was removed from the electrochemical cell, the Nafion membrane was separated from the Pt foils and washed with $0.5 \mathrm{M}$ $\mathrm{H}_{2} \mathrm{SO}_{4}$. It was found that anthranilic acid present in the portion of the Nafion film between the Pt foils alone was polymerized and the monomer present in the part of the Nafion immersed in the electrolyte did not polymerize (Fig. 1(c)).

Electrochemical polymerization of conducting polymers in Nafion has been carried out by coating an inert electrode with Nafion [13-15]. The inert metallic substrate acts as a current collector when dipped in a liquid electrolyte. It has also been reported [15] that a composite of Nafion-conducting polymer is deposited electrochemically on Pt electrode in a liquid electrolyte consisting of the Nafion suspension and the monomer. These studies involve polymerization in a liquid medium and the Nafion is present as a coating on an electrode. Thus, free-standing films of conducting polymer-Nafion cannot be prepared by these methods. In the present study, contrarily, polymerization of anthranilic acid took place in the portion of the Nafion membrane that was not in direct contact with $0.5 \mathrm{M} \mathrm{H}_{2} \mathrm{SO}_{4}$. As the Nafion is proton 


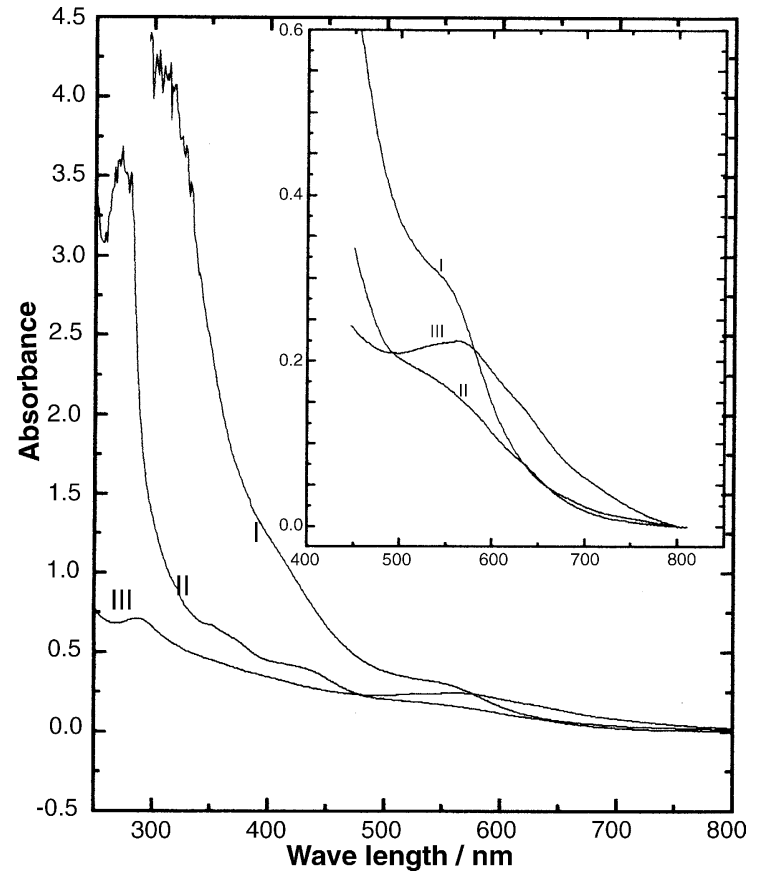

Fig. 2. UV-vis spectra of (I) PANA solution, (II) chemical PANA-Nafion membrane and (III) electrochemical PANA-Nafion membrane. The region between 800 and $400 \mathrm{~nm}$ is expanded and shown as the inset.

conducting electrolyte membrane, the un-sandwiched part (Fig. 1(b and c) marked (i)) acts as a junction between the liquid electrolyte and the solid electrolyte, that is, the portion of the Nafion membrane sandwiched between the Pt foils (Fig. 1 (b and c) marked (ii)). Interestingly, while anthranilic acid in un-sandwiched part does not undergo oxidation, there is formation of PANA only in the portion of the membrane between the Pt electrodes. It was found $\mathrm{O}_{2}$ evolution occurs instead of polymerization of anthranilic acid if the $\mathrm{Pt}$ foils of the WE assembly (Fig. 1(a)) were in contact with the $\mathrm{H}_{2} \mathrm{SO}_{4}$ electrolyte. By this novel electrochemical polymerization technique, it became possible to prepare free-standing PANA-Nafion membranes. In general, this procedure is expected to be useful for preparation of other conducting polymers in free-standing ionomeric membranes.

UV-vis spectra of a chemically polymerized PANANafion and an electrochemically polymerized PANA-Nafion are shown in Fig. 2. Spectrum of PANA solution in $0.5 \mathrm{M}$ $\mathrm{H}_{2} \mathrm{SO}_{4}$ is also shown for comparison. The peak observed at $270-300 \mathrm{~nm}$ is attributed to $\pi-\pi^{*}$ transition in benzenoid units [18] and the peak at $550-580 \mathrm{~nm}$ to exciton-like transition in quinoid diimino units [19]. Another absorbance band at $420-430 \mathrm{~nm}$ is also due to exciton-like transition in emeraldine salt form. The less intensity of the bands observed for PANA in comparison with the spectra of PANI is due to steric effect of carboxylic group [20], smaller chain length and confinement in Nafion membrane. The spectra of both chemical and electrochemical PANA-Nafion membranes resemble to the spectrum of PANA in solution thus providing evidence for the presence of PANA confined as a solid in the Nafion

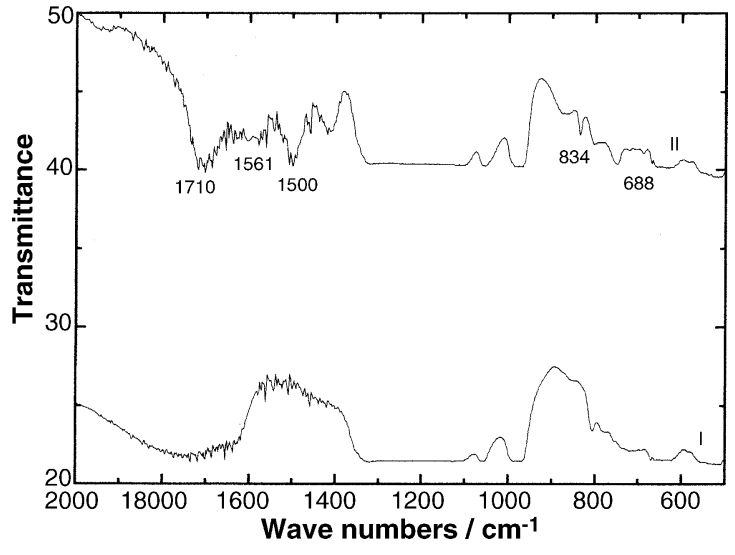

Fig. 3. FT-IR spectra of (I) bare Nafion membrane and (II) PANA-Nafion membrane.

membranes. Furthermore, the spectra of PANA prepared by chemical and electrochemical routes are almost identical. The exact band positions depend on several parameters such as chain length, the overall oxidation state of the polymer, interchain or intrachain charge-transfer, the extent of conjugation between adjacent phenyl rings, the steric effect of the carboxylate groups in the polymer chain, etc. [20]. The variations in band position of the spectra (Fig. 2) are likely to be due to variations in these parameters. The PANA-Nafion membranes were soaked in $0.5 \mathrm{M} \mathrm{H}_{2} \mathrm{SO}_{4}$ solution for a few days and UV-vis spectra were recorded again. The spectra were reproducible and the visual observation did not indicate any decrease in colour intensity due to soaking in the acidic solution. These observations suggest that PANA confined in the Nafion membrane does not undergo dissolution.

The confinement of PANA in Nafion membrane was further supported by recording FT-IR spectra (Fig. 3). The bands at $1200,1147,1056,981$ and $969 \mathrm{~cm}^{-1}$ are due to the characteristic functional groups in Nafion [17]. The additional bands at 1710, 1561, 1500, 834 and $688 \mathrm{~cm}^{-1}$ observed in the spectrum of PANA-Nafion are attributed to PANA [14]. The absorption band at $1710 \mathrm{~cm}^{-1}\left(1693 \mathrm{~cm}^{-1}\right.$ in [14]) is due to $\mathrm{C}=\mathrm{O}$ stretching, $1561 \mathrm{~cm}^{-1}\left(1575-1567 \mathrm{~cm}^{-1}\right.$ [21] is to benzenoid ring $\mathrm{C}=\mathrm{C}$ stretching and $834-688 \mathrm{~cm}^{-1}$ $\left(838-685 \mathrm{~cm}^{-1}\right.$ [21]) is to $\mathrm{C}-\mathrm{H}$ out of plane bending modes.

The electrochemical behaviour of PANI films on $\mathrm{Pt}$ substrate has been widely reported by recording cyclic voltammograms in acidic electrolytes [1]. The voltammograms are characterized by several current peaks corresponding to intrinsic redox processes of PANI. Among them, a pair of current peaks at about $0.2 \mathrm{~V}$ corresponding to the reversible transformation of leucomeraldine/emeraldine and another pair at about $0.7 \mathrm{~V}$ corresponding to emeraldine/pernigraniline reversible transition are prominent. A PANA-Nafion film was sandwiched between two Pt foils (Fig. 1( $\mathrm{a}$ and $\mathrm{b})$ ), dipped the un-polymerized part of the Nafion in an electrochemical cell consisting of $0.5 \mathrm{M} \mathrm{H}_{2} \mathrm{SO}_{4}$ and cyclic voltammograms were recorded at a sweep rate of $0.5 \mathrm{mV} \mathrm{s}^{-1}$. The voltammogram (Fig. 4) is characterized by a 


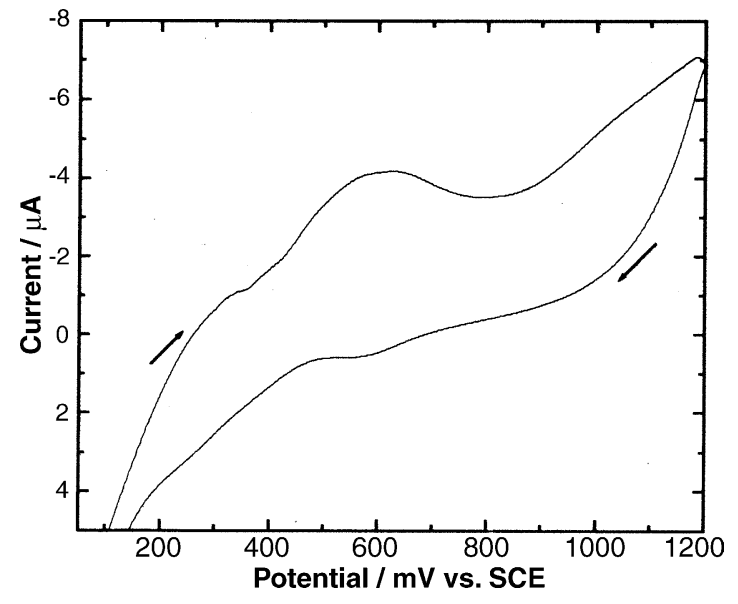

Fig. 4. Cyclic voltammogram of PANA-Nafion membrane. Area of each Pt foil used for Pt/Nafion/Pt sandwich is $1.25 \mathrm{~cm}^{2}$.

pair of broad current peaks in $0.5-0.6 \mathrm{~V}$ range, suggesting the electrochemical activity of PANA present inside the Nafion membrane. The nature of this voltammogram differs from the voltammogram of PANI films [1] because of the presence of - $\mathrm{COOH}$ group on PANI backbone, due to confinement in the Nafion pores and also due to the resistance of the membrane.

A PANA-Nafion membrane was examined for its optical properties in acidic $\left(0.5 \mathrm{M} \mathrm{H}_{2} \mathrm{SO}_{4}\right)$, neutral $\left(0.5 \mathrm{M} \mathrm{Na}_{2} \mathrm{SO}_{4}\right)$ and alkaline $(0.5 \mathrm{M} \mathrm{NaOH})$ media. The membrane was soaked in the respective solutions for about $2 \mathrm{~h}$ before recording UV-vis spectra and optical photographs. The photographs recorded for these membranes showed different colours, and the changes in colour were found reversible. That is, the membrane soaked in acidic medium was transferred to alkaline medium, and then back to the acidic solution. The original colour was restored in acidic medium. The UV-vis spectra (Fig. 5) suggested that the absorbance bands at $270-300,390-420$ and $560-570 \mathrm{~nm}$ were present for the membranes treated in solutions of a wide $\mathrm{pH}$ range. It is interesting to note that the $390-420$ band of PANI is

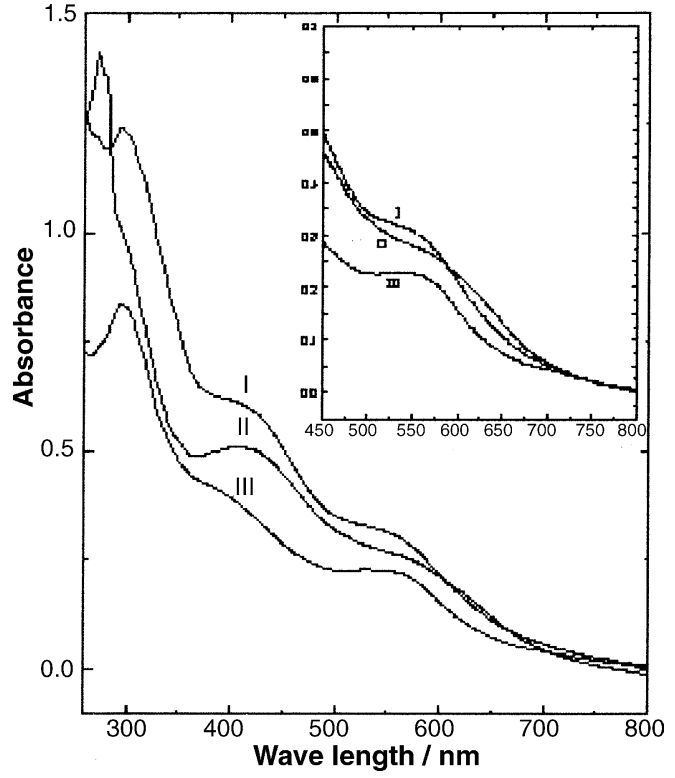

Fig. 5. UV-vis spectra of PANA-Nafion after treating in (I) $0.5 \mathrm{M} \mathrm{H}_{2} \mathrm{SO}_{4}$, (II) $1 \mathrm{M} \mathrm{Na}_{2} \mathrm{SO}_{4}$ and (III) $1 \mathrm{M} \mathrm{NaOH}$. The region between 800 and $400 \mathrm{~nm}$ is expanded and shown as the inset.

found only for $\mathrm{pH}<7$ and it disappears in alkaline solutions [22], whereas this absorbance band is clearly observed for PANA-Nafion in the present study. This is due to the presence of $-(\mathrm{COOH})$ group present on PANA chain. It is also due to the likely fact that $\mathrm{pH}$ inside the Nafion membrane could be lower than the surrounding solution. Electrochemically prepared PANA-Nafion membranes were examined by recording SEM micrographs (Fig. 6). The PANA crystallites are found in the shape of short, thick rods of about $2 \mu \mathrm{m}$ length and $0.75 \mu \mathrm{m}$ diameter occupying the voids present in the Nafion membrane. Similar SEM micrographs have been reported for a conducting polymer confined in polycarbonate membranes [23]. In this study, electrochemical polymerization of $o$-methoxyaniline has produced $100-400 \mathrm{~nm}$ size crystallites of poly(o-methoxyaniline) in polycarbonate membranes.
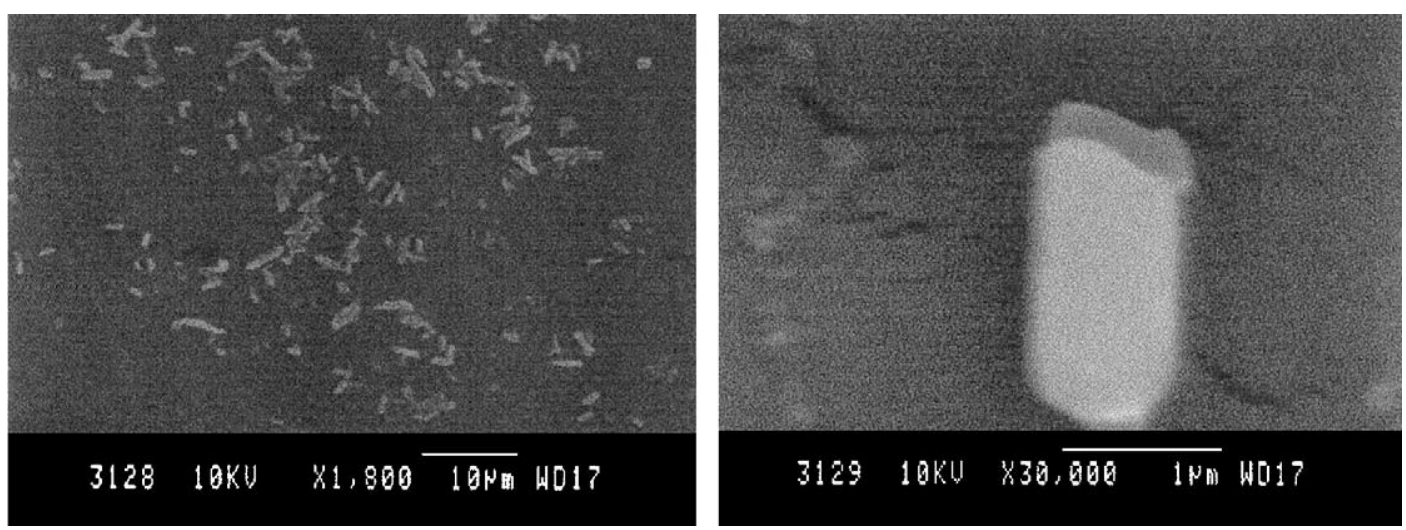

Fig. 6. SEM images of PANA-Nafion membrane in two magnifications. 


\section{Conclusion}

To prevent the solubility, poly(anthranilic acid) is confined in the cavities of the Nafion membrane by polymerizing anthranilic acid using a novel electrochemical as well as chemical methods. The PANA is characterized by electrochemical, optical, spectroscopic and scanning electron microscopic studies. It is anticipated that the solid form of poly(anthranilic acid) would be useful as a self-doped polymer for several application.

\section{Acknowledgement}

Authors thank Prof. A.K. Shukla and R.K. Raman for the Nafion samples.

\section{References}

[1] D.C. Trivedi, in: H.S. Nalwa (Ed.), Handbook of Organic Conductive Molecules and Polymers, vol. 2, Wiley, New York, 1997, p. 504.

[2] M.S.A. Abdou, S. Holdcroft, in: H.S. Nalwa (Ed.), Handbook of Organic Conductive Molecules and Polymers, vol. 2, Wiley, New York, 1997, p. 833.

[3] J. Yue, A.J. Esptein, J. Am. Chem. Soc. 112 (1990) 2800.

[4] J. Yue, Z.H. Wang, K.R. Cromack, A.J. Esptein, A.G. Macdiarmid, J. Am. Chem. Soc. 113 (1991) 2665.
[5] K. Krishnamoorthy, A.Q. Contractor, A. Kumar, Chem. Commun. (2002) 240

[6] S.M. Park, in: H.S. Nalwa (Ed.), Handbook of Organic Conductive Molecules and Polymers, vol. 3, Wiley, New York, 1997, p. 428.

[7] H. Yan, H.J. Wang, S. Adisasmito, N. Toshima, Bull. Chem. Soc. Jpn. 69 (1996) 2395.

[8] H.S.O. Chan, S.C. Ng, W.S. Sim, K.L. Tan, B.T.G. Tan, Macromolecules 25 (1992) 6029.

[9] L. Jiang, Q. Xie, L. Yang, X. Yang, S. Yao, J. Colloid Interface Sci. 274 (2004) 150.

[10] K.R. Prasad, N. Munichandraiah, J. Electrochem. Soc. 149 (2002) A1393.

[11] K.R. Prasad, N. Munichandraiah, Synth. Met. 123 (2001) 459.

[12] M. Fabrizio, G. Mengoli, M.M. Musiani, F. Paolucci, J. Electroanal. Chem. 300 (1991) 23.

[13] F.R.F. Fan, A.J. Bard, J. Electrochem. Soc. 133 (1986) 301

[14] R.M. Penner, C.R. Martin, J. Electrochem. Soc. 133 (1986) 310

[15] P. Aldebert, P. Audebert, M. Armand, G. Bidan, M. Pineri, J. Chem. Soc. Chem. Commun. (1986) 1636.

[16] K. Shigehara, M. Hara, A. Yamada, Synth. Met. 18 (1987) 721

[17] J. Liu, H. Wang, S. Cheng, K.Y. Chan, Chem. Commun. (2004) 728.

[18] W.B. Euler, Solid State Commun. 57 (1986) 857.

[19] S. Stafstrom, J.L. Bredas, A.J. Epstein, H.S. Woo, D.B. Tanner, W.S. Huang, A.G. Macdiarmid, Phys. Rev. Lett. 59 (1987) 1464.

[20] N. Toshima, H. Yan, Bull. Chem. Soc. Jpn. 68 (1995) 1056

[21] B.L. Rivas, C.O. Sanchez, J. Appl. Polym. Sci. 89 (2003) 2641.

[22] J. Stejskal, P. Kratochvil, N. Radhakrishnan, Synth. Met. 61 (1993) 225.

[23] M. Strawski, M.L. Donten, M. Donten, M. Szklarczyk, J. Solid State Electrochem. 8 (2004) 398. 